Article

\title{
Increased Serum Parathyroid Hormone, Osteocalcin and Alkaline Phosphatase Are Associated with a Long-Term Adverse Cardiovascular Outcome after Coronary Artery Bypass Graft Surgery
}

\author{
Olga Barbarash 1,2, Mikhail Zykov ${ }^{1}$, Vasiliy Kashtalap ${ }^{1,2}$, Oksana Hryachkova ${ }^{1}$, \\ Alexandr Kokov ${ }^{1}{ }^{(D}$, Olga Gruzdeva ${ }^{1}$, Irina Shibanova ${ }^{1}$ and Anton Kutikhin ${ }^{1, *}$ \\ 1 Research Institute for Complex Issues of Cardiovascular Diseases, Kemerovo 650002, Russia; \\ barbol@kemcardio.ru (O.B.); mvz83@mail.ru (M.Z.); kashvv@kemcardio.ru (V.K.); \\ hrychon@kemcardio.ru (O.H.); kokoan@kemcardio.ru (A.K.); gruzov@kemcardio.ru (O.G.); \\ shibia@kemcardio.ru (I.S.) \\ 2 Department of Cardiology and Cardiovascular Surgery, Kemerovo State Medical University, \\ Kemerovo 650056, Russia \\ * Correspondence: kytiag@kemcardio.ru; Tel.: +7-960-907-70-67
}

Received: 11 September 2019; Accepted: 5 October 2019; Published: 8 October 2019

\begin{abstract}
Despite the fact that an association of osteopoenia/osteoporosis with elevated risk of coronary artery calcification (CAC) and coronary atherosclerosis (CA) is well-established, it remains unclear whether bone turnover markers can be employed in long-term prognostication of such patients. Here we measured serum calcium, phosphate, calcitonin, parathyroid hormone (PTH), osteoprotegerin, osteocalcin, osteopontin, alkaline phosphatase and its bone isoenzyme, subsequently correlating them with an adverse cardiovascular outcome after 3 years of follow-up. The extent of brachiocephalic artery stenosis, CA, or CAC, as well as prevalence of osteopoenia/osteoporosis before the coronary artery bypass graft (CABG) surgery, did not differ between outcome groups, suggesting that subtle molecular mechanisms might be involved in determining the outcome rather than clinical or subclinical disease. After stepwise logistic regression, serum osteocalcin $>26.8 \mathrm{ng} / \mathrm{mL}$ and PTH $>$ $49.1 \mathrm{pg} / \mathrm{mL}$ were independent predictors of an adverse outcome. Serum ionised calcium correlated with multivessel coronary artery disease; moreover, patients with severe CA (SYNTAX score > 21) had higher serum ionised calcium than those with mild CA. Likewise, serum alkaline phosphatase was associated with severe CA and CAC (Agatston score > 400). In conclusion, serum PTH, osteocalcin, and alkaline phosphatase are associated with an adverse cardiovascular outcome 3 years after CABG surgery regardless of osteopoenia/osteoporosis, coronary/peripheral atherosclerosis, and CAC.
\end{abstract}

Keywords: osteopoenia; osteoporosis; coronary atherosclerosis; coronary artery disease; adverse outcome; bone turnover markers; ionised calcium; osteocalcin; parathyroid hormone; alkaline phosphatase

\section{Introduction}

Current advances in the treatment of coronary artery disease (CAD) are mainly related to myocardial revascularisation. Coronary artery bypass graft (CABG) surgery significantly improves prognosis and quality of life in all patients with CAD, particularly in those with high cardiovascular risk [1]. However, angina pectoris is a prevalent (around 25\%) long-term complication of CABG surgery due to a graft occlusion or coronary atherosclerosis (CA), both of which do frequently occur in comorbid patients; hence, screening for the markers of an adverse cardiovascular outcome is rapidly ongoing $[2,3]$. 
Coronary artery calcification (CAC) is a major risk factor of cardiovascular events and death in general population [4], as well as patients with chronic kidney disease (CKD) [5,6] or CAD [7]. Further, breast arterial calcification is also independently associated with an increased risk of all-cause and cardiovascular death [8]. In addition, CAC correlates with a lower efficacy of CABG surgery [9]. Extraskeletal calcification is to a large extent similar to bone tissue formation [10], yet its mechanisms are still under scrutiny. Despite osteopoenia/osteoporosis is associated with both CAC and CA [11], prognostic significance of bone turnover markers in patients with stable angina pectoris (SAP) after CABG surgery has been scarcely evaluated.

\section{Materials and Methods}

The study sample consisted of 111 consecutive male patients admitted to Research Institute for Complex Issues of Cardiovascular Diseases in 2015. Criteria of inclusion were CABG surgery due to CCS grade I-III SAP, age $<75$ years, and a written informed consent to participate in the study. Criteria of exclusion were progression of SAP to CCS grade IV, New York Heart Association class IV chronic heart failure, past medical history of CABG surgery, diagnosed acute/chronic liver or kidney failure, cancer, chronic obstructive pulmonary disease, autoimmune, endocrine (excluding type 2 diabetes mellitus), mental, blood, and/or severe digestive disorders, alcoholism, obesity (defined as body mass index $\geq 30 \mathrm{~kg} / \mathrm{m}^{2}$ ), and use of glucocorticoids $>3$ months all documented from the medical records. The investigation was carried out in accordance with the Good Clinical Practice and the Declaration of Helsinki. The study protocol was approved by the Local Ethical Committee of Research Institute for Complex Issues of Cardiovascular Diseases (ethical approval code 44025, approved on 12 December 2014). All patients provided written informed consent after receiving a full explanation of the study.

SAP, chronic heart failure, arterial hypertension, and type 2 diabetes mellitus were diagnosed according to the respective European Society of Cardiology guidelines [12-15] while overweight/obesity was defined as recommended in National Institute for Health and Care Excellence guidelines [16]. Glomerular filtration rate was calculated according to Modification of Diet in Renal Disease formula. Past medical history of MI, stroke, and smoking status were defined using the medical records.

Echocardiography (Sonos 2500, Hewlett Packard), color duplex screening of brachiocephalic arteries (Vivid 7 Dimension, General Electric Healthcare), coronary angiography (Innova 3100, General Electric Healthcare), multislice spiral computed tomography (SOMATOM Sensation 64 and Leonardo multimodality workstation, Siemens Healthcare), and dual-energy X-ray absorptiometry (Norland XR-46, Orthometrix) were performed in all patients before the CABG surgery. The results of coronary angiography and multislice spiral computed tomography were evaluated using SYNTAX score to measure the severity of $\mathrm{CA}$ and Agatston score to quantitate CAC, respectively. The results of dual-energy X-ray absorptiometry were interpreted according to the International Society for Clinical Densitometry Official Positions [17]. Bone mineral density of $\leq 1$ standard deviation below the mean peak bone mass (average of healthy young adult male) at the lumbar spine $\left(\mathrm{L}_{\mathrm{I}}-\mathrm{L}_{\mathrm{IV}}\right)$ and femoral neck was considered as normal. Osteopoenia and osteoporosis were defined as a bone mineral density of 1-2.49 and $\geq 2.5$ standard deviations below the mean peak bone mass, respectively. Clinicopathological features of the patients are indicated in Table 1.

During the hospital stay, all patients received the standard therapy of antiplatelet drugs, beta-blockers, angiotensin-converting enzyme inhibitors or angiotensin receptor II blockers, and lipid-lowering drugs which were also prescribed to them before hospital discharge. Follow-up was performed by an outpatient visit in 91 (82\%) patients; other individuals were unavailable due to the change of phone number or place of residence. Study endpoints included progression of AP, MI, or cardiovascular death.

Measurement of serum calcium, phosphate, and alkaline phosphatase was performed before the CABG surgery using the respective kits (Biosys Health) and KONELAB 320i biochemical analyzer (Thermo Scientific) (Table 2). Serum bone alkaline phosphatase, calcitonin, PTH, osteocalcin, osteoprotegerin, and osteopontin was quantified by an enzyme-linked immunosorbent assay using 
the respective kits of Metra Biosystems (8012), Biomerica (7024 and 7022), IDS (AC-11F1), Invitrogen (BMS2021INST), and Enzo Life Sciences (ADI-900-142) (Table 2).

Table 1. Clinicopathological features of the patients $(n=91)$.

\begin{tabular}{cc}
\hline Feature & Value \\
\hline Median age (interquartile range) & $61(55-65)$ \\
\hline Past medical history of myocardial infarction, $n(\%)$ & $70(76.9)$ \\
\hline Past medical history of stroke, $n(\%)$ & $6(6.6)$ \\
\hline Arterial hypertension, $n(\%)$ & $83(91.2)$ \\
\hline Median left ventricular ejection fraction, $\%$ (interquartile range) & $57(48-63)$ \\
\hline Type 2 diabetes mellitus, $n(\%)$ & $16(17.6)$ \\
\hline Median body mass index, $\mathrm{kg} / \mathrm{m}^{2}$ (interquartile range) & $24.8(27.5-29.4)$ \\
\hline Smoking, $n(\%)$ & $52(57.1)$ \\
\hline Bedian glomerular filtration rate, $\mathrm{mL} / \mathrm{min}^{\prime} / .73$ m ${ }^{2}$ (interquartile range) & $103(85-123)$ \\
\hline Median SYNTAX score (interquartile range) & $15(16.5)$ \\
\hline Median Agatston score (interquartile range) & $21.9(20.0-23.8)$ \\
\hline Osteopoenia, $n(\%)$ & $781(624-936)$ \\
\hline Osteoporosis, $n(\%)$ & $49(53.8)$ \\
\hline
\end{tabular}

Table 2. Biochemical parameters regulating mineral homeostasis measured before the CABG surgery $(n=91)$.

\begin{tabular}{ccc}
\hline Parameter & Median $\mathbf{( 9 5 \% \text { Confidence Interval) }}$ & Reference Values (Min-Max) \\
\hline Total calcium, $\mathrm{mmol} / \mathrm{L}$ & $2.30(2.25 ; 2.34)$ & $2.15-2.6$ \\
\hline Ionised calcium, $\mathrm{mmol} / \mathrm{L}$ & $0.94(0.92 ; 0.96)$ & $1.16-1.32$ \\
\hline Phosphate, $\mathrm{mmol} / \mathrm{L}$ & $0.95(0.92 ; 0.99)$ & $0.87-1.9$ \\
\hline Calcitonin, $\mathrm{pg} / \mathrm{mL}$ & $11.16(9.03 ; 13.29)$ & $<30$ \\
\hline Parathyroid hormone, $\mathrm{pg} / \mathrm{mL}$ & $31.97(24.18 ; 51.81)$ & $16-46$ \\
\hline Osteoprotegerin, $\mathrm{pg} / \mathrm{mL}$ & $134.47(114.61 ; 154.32)$ & $36.97-101.0$ \\
\hline Osteocalcin, $\mathrm{ng} / \mathrm{mL}$ & $21.96(19.29 ; 24.63)$ & $9.6-40.8$ \\
\hline Osteopontin, $\mathrm{ng} / \mathrm{mL}$ & $7.06(6.41 ; 7.71)$ & $0.26-30.0$ \\
\hline Alkaline phosphatase, $\mathrm{U} / \mathrm{L}$ & $126.63(109.44 ; 143.82)$ & $42-306$ \\
\hline Bone alkaline phosphatase, $\mathrm{U} / \mathrm{L}$ & $22.92(20.60 ; 25.23)$ & $15-41.3$ \\
\hline
\end{tabular}

Statistical analysis was carried out utilising SPSS 22 (IBM). A sampling distribution was assessed by Shapiro-Wilk test. Descriptive data were represented by proportions, median and interquartile range (25th and 75th percentiles). Intergroup differences were evaluated by Mann-Whitney U-test. To assess the correlation, Spearman's rank correlation coefficient, as well as enter and stepwise logistic regression, were employed. Threshold values for logistic regression equations were selected according to the highest quartile in patients having favorable outcome. $p$ values $\leq 0.05$ were regarded as statistically significant.

\section{Results}

We consecutively recruited 111 male patients who underwent CABG surgery due to Canadian Cardiovascular Society (CCS) grade I-III SAP; follow-up was carried out by an outpatient visit and was 
successful in 91/111 (82\%) patients. After 3 years of follow-up, 70 out of 91 (76.9\%) patients did not have any symptoms of CAD, while $16(17.6 \%)$ suffered from recurrent CCS class I-II SAP. Cardiovascular death was registered in $4(4.4 \%)$ patients due to myocardial infarction (MI) (3/4) or stroke (1/4) while 1 $(1.1 \%)$ patient experienced non-fatal MI. There were no differences in the extent of brachiocephalic artery stenosis, CA, or CAC as well as in prevalence of osteopoenia/osteoporosis before the CABG surgery in patients with favorable and adverse cardiovascular outcome (Table 3). Post-discharge treatment was also similar and included antiplatelet drugs, beta-blockers, angiotensin-converting enzyme inhibitors/angiotensin receptor II blockers, and lipid-lowering drugs.

Table 3. Color duplex screening, coronary angiography, and multislice spiral computed tomography results in relation to the adverse cardiovascular outcome after coronary artery bypass graft (CABG) surgery $(n=91)$.

\begin{tabular}{|c|c|c|c|}
\hline \multirow[b]{2}{*}{ Parameter } & \multicolumn{2}{|c|}{ Patient Groups } & \multirow[b]{2}{*}{$\mathbf{p}$} \\
\hline & $\begin{array}{c}\text { Favorable } \\
\text { Outcome }(n=70)\end{array}$ & $\begin{array}{l}\text { Adverse Outcome } \\
(n=21)\end{array}$ & \\
\hline Brachiocephalic artery stenosis $>50 \%, n(\%)$ & $12(17.1)$ & $3(14.3)$ & 0.75 \\
\hline $\begin{array}{l}\text { Multivessel coronary artery disease } \\
\qquad(3 \text { arteries affected }),(n, \%)\end{array}$ & $48(68.6)$ & $11(52.4)$ & 0.17 \\
\hline SYNTAX score $>32,(n, \%)$ & $17(24.3)$ & $7(33.3)$ & 0.41 \\
\hline Syntax score, median (interquartile range) & $24(16-32)$ & $28(13-34)$ & 0.57 \\
\hline Agatston score $>400,(n, \%)$ & $42(60.0)$ & $14(66.7)$ & 0.58 \\
\hline $\begin{array}{l}\text { Agatston score, median } \\
\text { (interquartile range) }\end{array}$ & $573(247-1088)$ & $630(206-984)$ & 0.78 \\
\hline Osteoporosis, $n(\%)$ & $19(27.1)$ & $7(33.3)$ & \multirow{2}{*}{0.80} \\
\hline Osteopoenia, $n(\%)$ & $39(55.7)$ & $10(47.6)$ & \\
\hline
\end{tabular}

Regarding the parameters of mineral homeostasis, adverse cardiovascular outcome was not associated with preoperative serum calcium, phosphate, calcitonin, or parathyroid hormone (PTH) (Table 4). Yet, patients with an adverse outcome had 2.2-, 1.5-, and 1.2-fold higher serum alkaline phosphatase, osteopontin, and osteocalcin along with 1.6-fold lower osteoprotegerin as compared to those with a favorable outcome (Table 4).

Table 4. Parameters of mineral homeostasis in relation to the adverse cardiovascular outcome upon CABG surgery.

\begin{tabular}{cccc}
\hline Parameter & $\begin{array}{c}\text { Favorable Outcome }(n=70) \\
\text { Median (Interquartile Range) }\end{array}$ & $\begin{array}{c}\text { Adverse Outcome }(n=21) \\
\text { Median (Interquartile Range) }\end{array}$ & $p$ \\
\hline Total calcium, mmol/L & $2.28(2.10-2.4)$ & $2.39(2.20-2.58)$ & 0.72 \\
\hline Ionised calcium, $\mathrm{mmol} / \mathrm{L}$ & $0.41(0.37-0.93)$ & $0.41(0.38-0.90)$ & 0.74 \\
\hline Phosphate, $\mathrm{mmol} / \mathrm{L}$ & $0.97(0.81-1.05)$ & $0.88(0.82-1.03)$ & 0.78 \\
\hline Calcitonin, $\mathrm{pg} / \mathrm{mL}$ & $8.51(6.98-12.57)$ & $7.80(6.79-8.83)$ & 0.14 \\
\hline Parathyroid hormone, $\mathrm{pg} / \mathrm{mL}$ & $32.62(25.09-49.07)$ & $45.64(21.81-62.18)$ & 0.42 \\
\hline Osteoprotegerin, $\mathrm{pg} / \mathrm{mL}$ & $113.58(61.54-183.92)$ & $70.40(51.74-109.13)$ & 0.02 \\
\hline Osteocalcin, $\mathrm{ng} / \mathrm{mL}$ & $18.77(12.67-22.47)$ & $22.75(16.40-37.66)$ & 0.01 \\
\hline Osteopontin, $\mathrm{ng} / \mathrm{mL}$ & $5.17(4.42-8.23)$ & $7.87(4.96-9.47)$ & 0.04 \\
\hline Alkaline phosphatase, $\mathrm{U} / \mathrm{L}$ & $73.5(49-183)$ & $165(81-218)$ & 0.03 \\
\hline Bone alkaline phosphatase, $\mathrm{U} / \mathrm{L}$ & $19.62(14.21-25.71)$ & $21.07(18.38-25.91)$ & 0.27 \\
\hline
\end{tabular}


To examine the differences in the parameters of mineral homeostasis in patient subgroups, we further stratified the study participants in relation to body mass index and type 2 diabetes mellitus. Similar to the patients with adverse outcome, those with overweight had 1.35-fold higher serum osteocalcin as compared to the subjects with normal body mass index (Table 5), while no statistically significant differences have been found in patients with or without type 2 diabetes mellitus (Table 6).

Table 5. Parameters of mineral homeostasis in relation to the patients with overweight (body mass index $\geq 25 \mathrm{~kg} / \mathrm{m}^{2}$ ).

\begin{tabular}{cccc}
\hline Parameter & $\begin{array}{c}\text { Without Overweight }(\boldsymbol{n = 6 9 )} \\
\text { Median (Interquartile Range) }\end{array}$ & $\begin{array}{c}\text { With Overweight }(\boldsymbol{n}=\mathbf{2 2}) \\
\text { Median (Interquartile Range) }\end{array}$ & $\boldsymbol{p}$ \\
\hline Total calcium, $\mathrm{mmol} / \mathrm{L}$ & $2.29(2.10-2.40)$ & $2.34(2.17-2.49)$ & 0.44 \\
\hline Ionised calcium, $\mathrm{mmol} / \mathrm{L}$ & $0.41(0.37-0.92)$ & $0.66(0.38-0.91)$ & 0.88 \\
\hline Phosphate, $\mathrm{mmol} / \mathrm{L}$ & $0.94(0.84-1.06)$ & $0.93(0.80-1.03)$ & 0.49 \\
\hline Calcitonin, $\mathrm{pg} / \mathrm{mL}$ & $8.28(6.94-11.26)$ & $9.0(7.03-13.81)$ & 0.29 \\
\hline Parathyroid hormone, $\mathrm{pg} / \mathrm{mL}$ & $32.62(24.79-52.33)$ & $38.36(23.59-54.83)$ & 0.88 \\
\hline Osteoprotegerin, $\mathrm{pg} / \mathrm{mL}$ & $103.73(60.52-181.66)$ & $88.88(58.28-128.58)$ & 0.39 \\
\hline Osteocalcin, $\mathrm{ng} / \mathrm{mL}$ & $18.29(12.83-26.78)$ & $24.67(18.70-35.07)$ & 0.01 \\
\hline Osteopontin, $\mathrm{ng} / \mathrm{mL}$ & $6.70(4.48-8.50)$ & $5.02(4.24-8.91)$ & 0.63 \\
\hline Alkaline phosphatase, $\mathrm{U} / \mathrm{L}$ & $117.0(56.0-212.0)$ & $88.0(61.0-179.0)$ & 0.69 \\
\hline Bone alkaline phosphatase, $\mathrm{U} / \mathrm{L}$ & $19.87(13.65-26.49)$ & $20.88(17.45-25.41)$ & 0.37 \\
\hline
\end{tabular}

Table 6. Parameters of mineral homeostasis in relation to the patients with type 2 diabetes mellitus.

\begin{tabular}{cccc}
\hline Parameter & $\begin{array}{c}\text { Without Type 2 Diabetes } \\
\text { Mellitus }(\boldsymbol{n}=\text { 75) Median } \\
\text { (Interquartile Range) }\end{array}$ & $\begin{array}{c}\text { With type 2 Diabetes } \\
\text { Mellitus }(\boldsymbol{n}=\mathbf{1 6}) \text { Median } \\
\text { (Interquartile Range) }\end{array}$ & $p$ \\
\hline Total calcium, $\mathrm{mmol} / \mathrm{L}$ & $2.27(2.10-2.48)$ & $2.31(2.09-2.44)$ & 0.93 \\
\hline Ionised calcium, $\mathrm{mmol} / \mathrm{L}$ & $0.41(0.37-0.91)$ & $0.63(0.38-0.95)$ & 0.63 \\
\hline Phosphate, $\mathrm{mmol} / \mathrm{L}$ & $0.93(0.81-1.05)$ & $1.0(0.83-1.04)$ & 0.64 \\
\hline Calcitonin, $\mathrm{pg} / \mathrm{mL}$ & $8.38(6.99-11.87)$ & $8.30(6.84-11.76)$ & 0.93 \\
\hline Parathyroid hormone, pg/mL & $36.27(24.49-55.91)$ & $30.38(21.81-33.91)$ & 0.08 \\
\hline Osteoprotegerin, $\mathrm{pg} / \mathrm{mL}$ & $101.54(59.50-180.29)$ & $80.40(52.62-171.15)$ & 0.62 \\
\hline Osteocalcin, $\mathrm{ng} / \mathrm{mL}$ & $20.76(15.55-31.69)$ & $15.01(12.60-21.30)$ & 0.09 \\
\hline Osteopontin, $\mathrm{ng} / \mathrm{mL}$ & $6.72(4.54-8.83)$ & $4.87(4.07-7.33)$ & 0.16 \\
\hline Alkaline phosphatase, $\mathrm{U} / \mathrm{L}$ & $100.0(60.0-208.0)$ & $130.0(40.5-175.0)$ & 0.44 \\
\hline Bone alkaline phosphatase, $\mathrm{U} / \mathrm{L}$ & $20.83(14.18-26.73)$ & $18.43(15.74-23.33)$ & 0.92 \\
\hline
\end{tabular}

Multivariate analysis confirmed that brachiocephalic artery stenosis, CA, CAC, and osteopoenia/ osteoporosis were not significant predictors of an adverse outcome in contrast to preoperative levels of serum osteopontin $>8.3 \mathrm{ng} / \mathrm{mL}, \mathrm{PTH}>49.1 \mathrm{pg} / \mathrm{mL}$, smoking, alkaline phosphatase $>186 \mathrm{U} / \mathrm{L}$, and osteocalcin $>26.8 \mathrm{ng} / \mathrm{mL}$, which were associated with a 76.1-, 27.8, 18.4, 17.7-, and 8.3-fold higher risk of adverse outcome, respectively (Table 7). Likewise, serum calcitonin $>8.8 \mathrm{pg} / \mathrm{mL}$ was associated with a 20-fold higher probability of a favorable outcome (Table 7). 
Table 7. Variables included into enter logistic regression equations to predict an adverse cardiovascular outcome.

\begin{tabular}{|c|c|c|c|c|c|c|c|}
\hline \multirow[b]{2}{*}{ Parameter } & \multirow{2}{*}{$\begin{array}{l}\text { Standardised } \\
\text { Regression } \\
\text { Coefficient (B) }\end{array}$} & \multirow{2}{*}{$\begin{array}{l}\text { Standard } \\
\text { Error }\end{array}$} & \multirow[b]{2}{*}{ Wald } & \multirow[b]{2}{*}{$p$} & \multirow{2}{*}{$\begin{array}{l}\text { Odds } \\
\text { Ratio }\end{array}$} & \multicolumn{2}{|c|}{$\begin{array}{l}95 \% \text { Confidence Interval } \\
\text { for the Odds Ratio }\end{array}$} \\
\hline & & & & & & $\begin{array}{l}\text { Lower } \\
\text { Bound }\end{array}$ & $\begin{array}{l}\text { Upper } \\
\text { Bound }\end{array}$ \\
\hline Age $>60$ years & -1.926 & 1.215 & 2.511 & 0.113 & 0.146 & 0.013 & 1.578 \\
\hline $\begin{array}{l}\text { Past medical history of } \\
\text { myocardial infarction }\end{array}$ & -2.912 & 1.447 & 4.053 & 0.044 & 0.054 & 0.003 & 0.926 \\
\hline Past medical history of stroke & 2.579 & 1.849 & 1.945 & 0.163 & 13.181 & 0.351 & 494.306 \\
\hline $\begin{array}{l}\text { New York Heart Association } \\
\text { class III-IV chronic heart failure }\end{array}$ & -0.558 & 1.288 & 0.188 & 0.665 & 0.572 & 0.046 & 7.140 \\
\hline $\begin{array}{l}\text { Left ventricular ejection fraction } \\
\qquad \geq 50 \%\end{array}$ & -2.160 & 1.441 & 2.247 & 0.134 & 0.115 & 0.007 & 1.943 \\
\hline Arterial hypertension & -1.657 & 1.843 & 0.808 & 0.369 & 0.191 & 0.005 & 7.068 \\
\hline Type 2 diabetes mellitus & -1.826 & 1.646 & 1.231 & 0.267 & 0.161 & 0.006 & 4.054 \\
\hline $\begin{array}{l}\text { Obesity (body mass index } \\
>30 \mathrm{~kg} / \mathrm{m}^{2} \text { ) }\end{array}$ & -0.979 & 1.012 & 0.936 & 0.333 & 0.376 & 0.052 & 2.730 \\
\hline Smoking & 2.914 & 1.451 & 4.033 & 0.045 & 18.437 & 1.073 & 316.902 \\
\hline $\begin{array}{l}\text { Brachiocephalic artery stenosis } \\
\qquad>50 \%\end{array}$ & 3.435 & 1.758 & 3.817 & 0.051 & 31.019 & 0.989 & 972.855 \\
\hline SYNTAX score $>32$ & 1.924 & 1.267 & 2.307 & 0.129 & 6.849 & 0.572 & 82.029 \\
\hline Agatston score $>1088$ & 1.045 & 1.093 & 0.913 & 0.339 & 2.843 & 0.333 & 24.231 \\
\hline Osteopoenia/osteoporosis & -1.978 & 1.327 & 2.223 & 0.136 & 0.138 & 0.010 & 1.864 \\
\hline Total calcium $>2.4 \mathrm{mmol} / \mathrm{L}$ & 2.461 & 1.276 & 3.719 & 0.054 & 11.719 & 0.961 & 142.986 \\
\hline Ionised calcium $>0.9 \mathrm{mmol} / \mathrm{L}$ & -1.901 & 1.556 & 1.494 & 0.222 & 0.149 & 0.007 & 3.151 \\
\hline Phosphate $>1.05 \mathrm{mmol} / \mathrm{L}$ & -1.708 & 1.305 & 1.714 & 0.190 & 0.181 & 0.014 & 2.338 \\
\hline Calcitonin $>8.8 \mathrm{pg} / \mathrm{mL}$ & -3.013 & 1.343 & 5.029 & 0.025 & 0.049 & 0.004 & 0.684 \\
\hline $\begin{array}{l}\text { Parathyroid hormone } \\
\quad>49.1 \mathrm{pg} / \mathrm{mL}\end{array}$ & 3.326 & 1.366 & 5.927 & 0.015 & 27.813 & 1.912 & 404.592 \\
\hline Osteoprotegerin $>61.5 \mathrm{pg} / \mathrm{mL}$ & -2.121 & 1.372 & 2.392 & 0.122 & 0.120 & 0.008 & 1.763 \\
\hline Osteocalcin $>26.8 \mathrm{ng} / \mathrm{mL}$ & 2.123 & 1.052 & 4.072 & 0.044 & 8.353 & 1.063 & 65.638 \\
\hline Osteopontin $>8.3 \mathrm{ng} / \mathrm{mL}$ & 4.332 & 1.879 & 5.313 & 0.021 & 76.082 & 1.912 & 3026.805 \\
\hline Alkaline phosphatase > $186 \mathrm{U} / \mathrm{L}$ & 2.979 & 1.437 & 4.298 & 0.038 & 17.669 & 1.177 & 328.778 \\
\hline $\begin{array}{l}\text { Bone alkaline phosphatase } \\
\qquad>25.9 \mathrm{U} / \mathrm{L}\end{array}$ & 3.431 & 1.557 & 4.855 & 0.068 & 0.032 & 0.002 & 0.685 \\
\hline Constant & 0.911 & 6.743 & 0.018 & 0.892 & 2.488 & & \\
\hline
\end{tabular}

After stepwise logistic regression analysis, serum osteocalcin $>26.8 \mathrm{ng} / \mathrm{mL}$ and PTH $>49.1 \mathrm{pg} / \mathrm{mL}$ were the only predictors that remained significant, with area under the ROC (Receiver operating characteristic) curve of 0.73 (95\% confidence interval 0.59-0.87) (Tables 8 and 9).

Positive correlations were found between serum ionised calcium and multivessel CAD $(r=0.58$, $p=0.01)$ and between serum phosphate and extent of CAC by means of Agatston score $(r=0.28$, $p=0.02$ ). In keeping with these findings, patients with severe CA (SYNTAX score $>21$ ) had higher serum ionised calcium $(0.86(0.38-0.92 \mathrm{mmol} / \mathrm{L})$ versus $0.39(0.36-0.87) \mathrm{mmol} / \mathrm{L}$, respectively, $p=0.018)$ and alkaline phosphatase (138.0 (60.0-209.0) versus $70.0(50.0-186.0) \mathrm{mmol} / \mathrm{L}$, respectively, $p=0.024)$ than those with lower SYNTAX score. Likewise, patients with severe CAC (Agatston score $>400$ ) were characterised by a higher serum alkaline phosphatase $(0.97(0.84-1.08)$ versus $0.88(0.80-1.03) \mathrm{mmol} / \mathrm{L}$, respectively, $p=0.01$ ) as compared with the individuals with mild calcification.

Therefore, preoperative serum concentrations of osteocalcin and PTH were reliably associated with an adverse cardiovascular outcome after 3 years of follow-up. Further, higher serum alkaline phosphatase positively correlated with severity of CA and CAC at the time of hospital admission. Serum 
ionised calcium and phosphate were also directly associated with severe CA and CAC, respectively, at the same time point.

Table 8. Significant variables predicting an adverse cardiovascular outcome in stepwise logistic regression analysis.

\begin{tabular}{|c|c|c|c|c|c|c|c|c|}
\hline & \multirow[b]{2}{*}{ Parameter } & \multirow{2}{*}{$\begin{array}{l}\text { Standardised } \\
\text { Regression } \\
\text { Coefficient (B) }\end{array}$} & \multirow[b]{2}{*}{$\begin{array}{c}\text { Standard } \\
\text { Error }\end{array}$} & \multirow[b]{2}{*}{ Wald } & \multirow[b]{2}{*}{$P$} & \multirow{2}{*}{$\begin{array}{l}\text { Odds } \\
\text { Ratio }\end{array}$} & \multicolumn{2}{|c|}{$\begin{array}{l}95 \% \text { Confidence Interval } \\
\text { for the Odds Ratio }\end{array}$} \\
\hline & & & & & & & $\begin{array}{l}\text { Lower } \\
\text { Bound }\end{array}$ & $\begin{array}{l}\text { Upper } \\
\text { Bound }\end{array}$ \\
\hline \multirow{2}{*}{ के } & $\begin{array}{l}\text { Parathyroid hormone } \\
\quad>49.1 \mathrm{pg} / \mathrm{mL}\end{array}$ & 1.312 & 0.521 & 6.327 & 0.012 & 3.712 & 1.336 & 10.317 \\
\hline & Constant & -2.998 & 0.792 & 14.325 & 0.000 & 0.050 & & \\
\hline \multirow{3}{*}{$\begin{array}{l}N \\
\text { चे } \\
\text { के }\end{array}$} & $\begin{array}{c}\text { Osteocalcin } \\
>26.8 \mathrm{ng} / \mathrm{mL}\end{array}$ & 1.142 & 0.549 & 4.331 & 0.037 & 3.132 & 1.069 & 9.181 \\
\hline & $\begin{array}{l}\text { Parathyroid hormone } \\
\quad>49.1 \mathrm{pg} / \mathrm{mL}\end{array}$ & 1.393 & 0.543 & 6.577 & 0.010 & 4.028 & 1.389 & 11.682 \\
\hline & Constant & -4.657 & 1.204 & 14.962 & 0.000 & 0.009 & & \\
\hline
\end{tabular}

Table 9. Prevalence of an adverse cardiovascular outcome and odds ratios in relation to the combinations of risk factors $(\mathrm{p}=0.00007)$.

\begin{tabular}{cccc}
\hline Risk Groups & $n$ & $\begin{array}{c}\text { Prevalence of Adverse } \\
\text { Cardiovascular Outcome }\end{array}$ & $\begin{array}{c}\text { Odds Ratio (95\% } \\
\text { Confidence Interval) }\end{array}$ \\
\hline $\begin{array}{c}\text { No increase in parathyroid hormone } \\
\text { and osteocalcin }\end{array}$ & 45 & $13.33 \%$ & $0.32(0.11-0.92)$ \\
\hline $\begin{array}{c}\text { Increase in either parathyroid hormone } \\
\text { or osteocalcin }\end{array}$ & 38 & $23.68 \%$ & $1.06(0.40-2.84)$ \\
\hline $\begin{array}{c}\text { Increase in both parathyroid hormone }> \\
49.1 \mathrm{pg} / \mathrm{mL} \text { and osteocalcin }>26.5 \mathrm{ng} / \mathrm{mL}\end{array}$ & 8 & $75.0 \%$ & $13.60(2.50-74.09)$ \\
\hline
\end{tabular}

\section{Discussion}

In the present study, we show that the parameters of mineral homeostasis, in particular osteocalcin, $\mathrm{PTH}$, and alkaline phosphatase, can be useful in prognostication after CABG surgery. Previously, serum PTH $>65 \mathrm{pg} / \mathrm{mL}$ was found associated with cardiovascular events in patients with chronic kidney disease (CKD) stages 3 and 4 [18] and with progression of CKD as well as mortality in those with CKD stages 2-4 [19]. Moreover, serum PTH $>6.8 \mathrm{pmol} / \mathrm{L}$ was an independent risk factor of CAD in a population-based cross-sectional Tromso study [20].

PTH evinces its effects through PTH 1 receptor which is expressed in cardiomyocytes, vascular smooth muscle cells, and endothelial cells and which level is increased during myocardial ischemia, cardiac fibrosis, and aging [21]. Elevation of serum PTH causes left ventricular hypertrophy [22], myocyte/capillary mismatch [23], and cardiac fibrosis [24]. Excessive PTH disrupts energetic metabolism in cardiomyocytes, leading to a calcium overload [25] and induces carbohydrate metabolism disorders [26] and vascular inflammation [27], correlating with a multivessel CAD, low left ventricular ejection fraction [28], and CAC in patients without CKD [29].

Osteocalcin is a pleiotropic protein regulating glucose and lipid metabolism, nitric oxide release, and endothelial homeostasis depending on its carboxylation status [30]. Opposite to $\mathrm{PTH}$, an association of serum osteocalcin with cardiovascular death is unclear, being U-shaped as well as gender- and age-dependent [31-33]. Data regarding the prognostic significance of alkaline phosphatase are also contradictory, varying between revascularisation modalities [34-36]. Albeit serum alkaline phosphatase may be also increased in patients with stroke, chronic heart failure, arterial hypertension, type 2 diabetes mellitus, and obesity, a number of studies showed its usefulness as a prognostic indicator 
during the long-term follow-up of patients with coronary artery disease [35,36] including those with ST-segment elevation MI [37]. In addition, SAP of CCS grade IV, New York Heart Association class IV chronic heart failure, and obesity were among the criteria of exclusion in our study.

Notably, we found bone turnover markers such as PTH, osteocalcin, and alkaline phosphatase associated with an adverse cardiovascular outcome after CABG surgery at a 3-year time point regardless of osteopoenia/osteoporosis, coronary or peripheral atherosclerosis, and CAC. Despite the fact that all indicated proteins are to a certain extent involved in bone resorption, atherosclerosis, and extraskeletal calcification, their predictive value possibly does not depend on respective clinical conditions, rather it is reflective of their combination at a subclinical level. Possible reasons for the discrepancies between the studies include sample size, duration of follow-up, and treatment regimens. We suggest that the preoperative screening of bone turnover markers can be useful in prognostication after CABG surgery due to SAP; however, additional studies on larger samples are necessary for the validation of this approach.

Author Contributions: Conceptualization, O.B., M.Z. and V.K.; Data curation, I.S.; Formal analysis, O.B., M.Z., V.K. and A.K. (Anton Kutikhin); Funding acquisition, O.B.; Investigation, M.Z., V.K., O.H., A.K. (Alexandr Kokov), O.G. and A.K. (Anton Kutikhin); Methodology, M.Z., V.K., A.K. (Alexandr Kokov) and O.G.; Project administration, O.B.; Resources, O.H., A.K. (Alexandr Kokov) and O.G.; Supervision, O.B.; Validation, O.B.; Writing—original draft, M.Z.; Writing一review \& editing, O.B., V.K. and A.K. (Anton Kutikhin)

Funding: This study was supported by the Complex Program of Basic Research under the Siberian Branch of the Russian Academy of Sciences within the Basic Research Topic of Research Institute for Complex Issues of Cardiovascular Diseases № 0546-2015-0011 “Pathogenetic basis for the development of cardiovascular implants from biocompatible materials using patient-oriented approach, mathematical modeling, tissue engineering, and genomic predictors".

Conflicts of Interest: The authors declare no conflict of interest. The funders had no role in the design of the study; in the collection, analyses, or interpretation of data; in the writing of the manuscript, or in the decision to publish the results.

\section{Abbreviations}

$\begin{array}{ll}\text { CAC } & \text { Coronary artery calcification } \\ \text { CA } & \text { Coronary atherosclerosis } \\ \text { PTH } & \text { Parathyroid hormone } \\ \text { CABG } & \text { Coronary artery bypass graft } \\ \text { SYNTAX } & \text { Synergy between Percutaneous Coronary Intervention with Taxus and Cardiac Surgery } \\ \text { CAD } & \text { Coronary artery disease } \\ \text { CKD } & \text { Chronic kidney disease } \\ \text { SAP } & \text { Stable angina pectoris } \\ \text { CCS } & \text { Canadian Cardiovascular Society } \\ \text { MI } & \text { Myocardial infarction } \\ \text { ROC } & \text { Receiver operating characteristic }\end{array}$

\section{References}

1. Doenst, T.; Haverich, A.; Serruys, P.; Bonow, R.O.; Kappetein, P.; Falk, V.; Velazquez, E.; Diegeler, A.; Sigusch, H. PCI and CABG for Treating Stable Coronary Artery Disease: JACC Review Topic of the Week. J. Am. Coll. Cardiol. 2019, 73, 964-976. [CrossRef] [PubMed]

2. Adelborg, K.; Horváth-Puhó, E.; Schmidt, M.; Munch, T.; Pedersen, L.; Nielsen, P.H.; Bøtker, H.E.; Toft Sørensen, H. Thirty-Year Mortality After Coronary Artery Bypass Graft Surgery: A Danish Nationwide Population-Based Cohort Study. Circ. Cardiovasc. Qual. Outcomes 2017, 10, e002708. [CrossRef]

3. Kikuchi, K.; Mori, M. Minimally invasive coronary artery bypass grafting: A systematic review. Asian Cardiovasc. Thorac. Ann. 2017, 25, 364-370. [CrossRef] [PubMed]

4. Kelkar, A.A.; Schultz, W.M.; Khosa, F.; Schulman-Marcus, J.; O’Hartaigh, B.W.; Gransar, H.; Blaha, M.J.; Knapper, J.T.; Berman, D.S.; Quyyumi, A.; et al. Long-Term Prognosis After Coronary Artery Calcium Scoring Among Low-Intermediate Risk Women and Men. Circ. Cardiovasc Imaging 2016, 9, e003742. [CrossRef] [PubMed] 
5. Górriz, J.L.; Molina, P.; Cerverón, M.J.; Vila, R.; Bover, J.; Nieto, J.; Barril, G.; Martínez-Castelao, A.; Fernández, E.; Escudero, V.; et al. Vascular calcification in patients with nondialysis CKD over 3 years. Clin. J. Am. Soc. Nephrol. 2015, 10, 654-666. [CrossRef]

6. Chen, J.; Budoff, M.J.; Reilly, M.P.; Yang, W.; Rosas, S.E.; Rahman, M.; Zhang, X.; Roy, J.A.; Lustigova, E.; Nessel, L.; et al. CRIC Investigators. Coronary Artery Calcification and Risk of Cardiovascular Disease and Death Among Patients With Chronic Kidney Disease. JAMA Cardiol. 2017, 2, 635-643. [CrossRef]

7. Wang, R.; Liu, X.; Wang, C.; Ye, X.; Xu, X.; Yang, C. Higher coronary artery calcification score is associated with adverse prognosis in patients with stable angina pectoris. J. Thorac. Dis. 2017, 9, 582-589. [CrossRef] [PubMed]

8. Hendriks, E.J.; Beulens, J.W.; de Jong, P.A.; van der Schouw, Y.T.; Sun, W.N.; Wright, C.M.; Criqui, M.H.; Allison, M.A.; Ix, J.H. Calcification of the splenic, iliac, and breast arteries and risk of all-cause and cardiovascular mortality. Atherosclerosis 2017, 259, 120-127. [CrossRef]

9. Ertelt, K.; Généreux, P.; Mintz, G.S.; Reiss, G.R.; Kirtane, A.J.; Madhavan, M.V.; Fahy, M.; Williams, M.R.; Brener, S.J.; Mehran, R.; et al. Impact of the severity of coronary artery calcification on clinical events in patients undergoing coronary artery bypass grafting (from the Acute Catheterization and Urgent Intervention Triage Strategy Trial). Am. J. Cardiol. 2013, 112, 1730-1737. [CrossRef]

10. Chan, J.J.; Cupples, L.A.; Kiel, D.P.; O’Donnell, C.J.; Hoffmann, U.; Samelson, E.J. QCT Volumetric Bone Mineral Density and Vascular and Valvular Calcification: The Framingham Study. J. Bone Miner. Res. 2015, 30, 1767-1774. [CrossRef]

11. Barbarash, O.L.; Lebedeva, N.B.; Kokov, A.N.; Novitskaya, A.A.; Hryachkova, O.N.; Voronkina, A.V.; Raskina, T.A.; Kashtalap, V.V.; Kutikhin, A.G.; Shibanova, I.A. Decreased Cathepsin K Plasma Level may Reflect an Association of Osteopoenia/Osteoporosis with Coronary Atherosclerosis and Coronary Artery Calcification in Male Patients with Stable Angina. Heart Lung Circ. 2016, 25, 691-697. [CrossRef] [PubMed]

12. Task Force Members; Montalescot, G.; Sechtem, U.; Achenbach, S.; Andreotti, F.; Arden, C.; Budaj, A.; Bugiardini, R.; Crea, F.; Cuisset, T.; et al. 2013 ESC guidelines on the management of stable coronary artery disease: The Task Force on the management of stable coronary artery disease of the European Society of Cardiology. Eur. Heart J. 2013, 34, 2949-3003. [CrossRef] [PubMed]

13. McMurray, J.J.; Adamopoulos, S.; Anker, S.D.; Auricchio, A.; Böhm, M.; Dickstein, K.; Falk, V.; Filippatos, G.; Fonseca, C.; Gomez-Sanchez, M.A.; et al. ESC guidelines for the diagnosis and treatment of acute and chronic heart failure 2012: The Task Force for the Diagnosis and Treatment of Acute and Chronic Heart Failure 2012 of the European Society of Cardiology. Developed in collaboration with the Heart Failure Association (HFA) of the ESC. Eur. J. Heart Fail. 2012, 14, 803-869. [CrossRef] [PubMed]

14. Mancia, G.; Fagard, R.; Narkiewicz, K.; Redon, J.; Zanchetti, A.; Böhm, M.; Christiaens, T.; Cifkova, R.; De Backer, G.; Dominiczak, A.; et al. 2013 ESH/ESC guidelines for the management of arterial hypertension: The Task Force for the Management of Arterial Hypertension of the European Society of Hypertension (ESH) and of the European Society of Cardiology (ESC). Eur. Heart J. 2013, 34, 2159-2219. [CrossRef] [PubMed]

15. Authors/Task Force Members; Rydén, L.; Grant, P.J.; Anker, S.D.; Berne, C.; Cosentino, F.; Danchin, N.; Deaton, C.; Escaned, J.; Hammes, H.P.; et al. ESC Guidelines on diabetes, pre-diabetes, and cardiovascular diseases developed in collaboration with the EASD: The Task Force on diabetes, pre-diabetes, and cardiovascular diseases of the European Society of Cardiology (ESC) and developed in collaboration with the European Association for the Study of Diabetes (EASD). Eur. Heart J. 2013, 34, 3035-3087. [CrossRef]

16. National Clinical Guideline Centre (UK). Obesity: Identification, Assessment and Management of Overweight and Obesity in Children. Young People and Adults: Partial Update of CG43; National Institute for Health and Care Excellence: London, UK, 2014.

17. Schousboe, J.T.; Shepherd, J.A.; Bilezikian, J.P.; Baim, S. Executive summary of the 2013 International Society for Clinical Densitometry Position Development Conference on bone densitometry. J. Clin. Densitom. 2013, 16, 455-466. [CrossRef] [PubMed]

18. Bhuriya, R.; Li, S.; Chen, S.C.; McCullough, P.A.; Bakris, G.L. Plasma parathyroid hormone level and prevalent cardiovascular disease in CKD stages 3 and 4: An analysis from the Kidney Early Evaluation Program (KEEP). Am. J. Kidney Dis. 2009, 53, S3-S10. [CrossRef] [PubMed]

19. Chartsrisak, K.; Vipattawat, K.; Assanatham, M.; Nongnuch, A.; Ingsathit, A.; Domrongkitchaiporn, S.; Sumethkul, V.; Distha-Banchong, S. Mineral metabolism and outcomes in chronic kidney disease stage 2-4 patients. BMC Nephrol. 2013, 14, 14. [CrossRef] [PubMed] 
20. Kamycheva, E.; Sundsfjord, J.; Jorde, R. Serum parathyroid hormone levels predict coronary heart disease: The Tromsø Study. Eur. J. Cardiovasc. Prev. Rehabil. 2004, 11, 69-74. [CrossRef]

21. Monego, G.; Arena, V.; Pasquini, S.; Stigliano, E.; Fiaccavento, R.; Leone, O.; Arpesella, G.; Potena, L.; Ranelletti, F.O.; Di Nardo, P.; et al. Ischemic injury activates PTHrP and PTH1R expression in human ventricular cardiomyocytes. Basic Res. Cardiol. 2009, 104, 427-434. [CrossRef] [PubMed]

22. Saleh, F.N.; Schirmer, H.; Sundsfjord, J.; Jorde, R. Parathyroid hormone and left ventricular hypertrophy. Eur. Heart J. 2003, 24, 2054-2060. [CrossRef] [PubMed]

23. Amann, K.; Breitbach, M.; Ritz, E.; Mall, G. Myocyte/capillary mismatch in the heart of uremic patients. J. Am. Soc. Nephrol. 1998, 9, 1018-1022. [PubMed]

24. Tyralla, K.; Amann, K. Morphology of the heart and arteries in renal failure. Kidney Int. Suppl. 2003, 84, S80-S83. [CrossRef] [PubMed]

25. Nishimura, M.; Tsukamoto, K.; Tamaki, N.; Kikuchi, K.; Iwamoto, N.; Ono, T. Risk stratification for cardiac death in hemodialysis patients without obstructive coronary artery disease. Kidney Int. 2011, 79, 363-371. [CrossRef] [PubMed]

26. Reis, J.P.; von Mühlen, D.; Kritz-Silverstein, D.; Wingard, D.L.; Barrett-Connor, E.; Vitamin, D. Parathyroid hormone levels and the prevalence of metabolic syndrome in community-dwelling older adults. Diabetes Care 2007, 30, 1549-1555. [CrossRef] [PubMed]

27. Martín-Ventura, J.L.; Ortego, M.; Esbrit, P.; Hernández-Presa, M.A.; Ortega, L.; Egido, J. Possible role of parathyroid hormone-related protein as a proinflammatory cytokine in atherosclerosis. Stroke 2003, 34, 1783-1789. [CrossRef]

28. Shekarkhar, S.; Foroughi, M.; Moatamedi, M.; Gachkar, L. The association of serum parathyroid hormone and severity of coronary artery diseases. Coron. Artery Dis. 2014, 25, 339-342. [CrossRef]

29. Wu, G.Y.; Xu, B.D.; Wu, T.; Wang, X.Y.; Wang, T.X.; Zhang, X.; Wang, X.; Xia, Y.; Zong, G.J. Correlation between serum parathyroid hormone levels and coronary artery calcification in patients without renal failure. Biomed. Rep. 2016, 5, 601-606. [CrossRef]

30. Tacey, A.; Qaradakhi, T.; Brennan-Speranza, T.; Hayes, A.; Zulli, A.; Levinger, I. Potential Role for Osteocalcin in the Development of Atherosclerosis and Blood Vessel Disease. Nutrients 2018, 10, E1426. [CrossRef]

31. Yeap, B.B.; Chubb, S.A.; Flicker, L.; McCaul, K.A.; Ebeling, P.R.; Hankey, G.J.; Beilby, J.P.; Norman, P.E. Associations of total osteocalcin with all-cause and cardiovascular mortality in older men. The Health In Men Study. Osteoporos. Int. 2012, 23, 599-606. [CrossRef]

32. Holvik, K.; van Schoor, N.M.; Eekhoff, E.M.; den Heijer, M.; Deeg, D.J.; Lips, P.; de Jongh, R. Plasma osteocalcin levels as a predictor of cardiovascular disease in older men and women: A population-based cohort study. Eur. J. Endocrinol. 2014, 171, 161-170. [CrossRef] [PubMed]

33. Zhang, X.; Shen, Y.; Xu, Y.; Xiong, Q.; Lu, Z.; Ma, X.; Bao, Y.; Jia, W. Association of serum osteocalcin levels with major adverse cardiovascular events: A 4.4-year retrospective cohort study. Clin. Exp. Pharmacol. Physiol. 2018, 45, 3-9. [CrossRef] [PubMed]

34. Kim, T.K.; Hong, D.M.; Choi, Y.H.; Koo, C.H.; Cho, Y.J.; Park, J.B.; Park, K.W.; Kim, H.S.; Jeon, Y. Preoperative Serum Alkaline Phosphatase and Clinical Outcome of Off-Pump Coronary Artery Bypass Surgery. Circ. J. 2017, 81, 799-805. [CrossRef] [PubMed]

35. Park, J.B.; Kang, D.Y.; Yang, H.M.; Cho, H.J.; Park, K.W.; Lee, H.Y.; Kang, H.J.; Koo, B.K.; Kim, H.S. Serum alkaline phosphatase is a predictor of mortality.; myocardial infarction, or stent thrombosis after implantation of coronary drug-eluting stent. Eur. Heart J. 2013, 34, 920-931. [CrossRef] [PubMed]

36. Ndrepepa, G.; Xhepa, E.; Braun, S.; Cassese, S.; Fusaro, M.; Schunkert, H.; Kastrati, A. Alkaline phosphatase and prognosis in patients with coronary artery disease. Eur. J. Clin. Investg. 2017, 47, 378-387. [CrossRef] [PubMed]

37. Oh, P.C.; Lee, K.; Kim, T.H.; Moon, J.; Park, H.W.; Jang, H.J.; Park, S.D.; Kwon, S.W.; Suh, J.; Kang, W.C. Prognostic impact of alkaline phosphatase measured at time of presentation in patients undergoing primary percutaneous coronary intervention for ST-segment elevation myocardial infarction. PLoS ONE 2017, 12, e0171914. [CrossRef]

(C) 2019 by the authors. Licensee MDPI, Basel, Switzerland. This article is an open access article distributed under the terms and conditions of the Creative Commons Attribution (CC BY) license (http://creativecommons.org/licenses/by/4.0/). 